\title{
Métaux et réglementation hospitalière
}

\author{
Metals and hospital regulation
}

\section{Élise TOGUYENI (1), Florence OU-CEBRON ${ }^{(1)}$, Christophe BERNERON(1), Betty DEHON $^{(2)}$, Michel LHERMITTE ${ }^{(2)}$}

(1) Pharmacie Centrale, CHRU de Lille, rue Philippe Marache - 59037 LILLE Cedex

(2) Laboratoire de Biochimie et de Biologie Moléculaire, Hôpital Calmette, CHRU de Lille, 2, avenue Oscar Lambret - 59037 LILLE Cedex

(Reçu le 10 mai 2001 ; accepté le 20 juin 2001)

\section{RÉSUMÉ}

La qualité de l'eau distribuée dans un établissement hospitalier doit répondre au minimum aux normes de potabilité définies par le décret 89-3 modifié et la directive européenne 98/83/CE.

Les métaux font partie des nombreux paramètres surveillés. Pour des usages particuliers comme l'hémodialyse, l'eau du réseau subit divers traitements afin de la rendre conforme aux spécifications de la Pharmacopée européenne.

Les conditions de prélèvements, d'acheminement et de conservation des échantillons, avant analyse, peuvent influer sur le résultat final.

Le dosage des principaux métaux dans l'eau, fait surtout appel à des méthodes basées sur la spectroscopie d'absorption ou d'émission atomique.

\section{SUMMARY}

The quality of water inside hospitable establishment system must at least, agree with standards of the modified ordinance 89-3 and the european directive for drinking water. Many parameters including the metals are supervised. For specific uses such as hemodialysis, several treatments are applied to obtain water with a quality according to specifications of european Pharmacopoeia.

Before analysis, the modalities of sampling, conveyance and conservation can modify the results. Various methods, such as atomic absorption or emission spectroscopy are used for the dosage of metals in water. 


\section{Introduction}

La composition d'une eau dépend de plusieurs paramètres : le secteur géographique, les évolutions saisonnières, climatiques, l'importance de la pollution humaine et industrielle ainsi que de son origine : source profonde ou source superficielle (1).

Les eaux destinées à la consommation humaine doivent satisfaire aux exigences de qualité définies à l'annexe I1 du décret n ${ }^{\circ} 89-3$ modifié du 3 janvier 1989 ainsi qu'à la directive européenne $n^{\circ} 98 / 83 / \mathrm{CE}$ du 3 novembre $1998(2,3)$.

Tout concessionnaire d'une distribution d'eau potable est tenu de faire vérifier la qualité de l'eau qui fait l'objet de cette distribution. Selon la taille de l'établissement, un hôpital consomme de 500 à 1200 litres d'eau par jour (4).

L' eau du réseau intérieur d'un établissement hospitalier provient : - soit d'une ressource privée (utilisation soumise à l'autorisation du Préfet), - soit du réseau d'adduction publique.

Les réseaux internes sont souvent très complexes. I1 appartient au Directeur d'établissement de garantir la potabilité de l'ensemble des points d'eau $(4,5)$.

La vérification de la qualité de l'eau comporte des analyses physico-chimiques et microbiologiques (près de 62 critères) $(4,6)$.

Les auteurs rapportent les normes de qualité et les méthodes de dosage applicables aux principaux métaux présents: - (i) dans l'eau du réseau intérieur d'un établissement de santé, - mais aussi (ii) dans une eau ayant subi divers traitements et destinée à un usage thérapeutique telle que l'eau pour dilution des solutions concentrées pour hémodialyse.

\section{Rappels réglementaires sur la qualité de l'eau des éta- blissements de santé}

L'eau à l'hôpital se subdivise en 2 grands réseaux : l'eau froide à usage multiple et l'eau chaude sanitaire.

\section{L'eau froide a de multiples usages :}

- sanitaire (hygiène des malades, entretien des locaux), - alimentaire (cuisine, eau de boisson, eau des fontaines réfrigérées),

- technique (lutte anti-incendie, traitement de l'air), - médical (balnéation, désinfection des matériels médico-chirurgicaux, hémodialyse),

- autres (blanchisseries, laboratoires, stérilisation)

Cette eau doit répondre aux normes de potabilité fixées par le décret n89-3 modifié relatif aux eaux destinées à la consommation humaine à l'exclusion des eaux minérales naturelles et par la directive européenne $n^{\circ} 98 / 83 / C E$ relative à la qualité des eaux destinées à la consommation humaine. Cette directive est en cours de transposition en droit français (7).

Le délai de mise en conformité de la qualité de l'eau pour les états membres, est de 5 ans à partir de son entrée en vigueur (3).

On relève quelques différences entre : le décret et la directive

- pour le cuivre, la norme passe de $1 \mathrm{mg} / \mathrm{l}$ à $2 \mathrm{mg} / \mathrm{l}$

- pour le nickel, de $50 \mu \mathrm{g} / \mathrm{l}$ à $20 \mu \mathrm{g} / 1$

- pour le plomb, de $50 \mu \mathrm{g} / \mathrm{l}$ actuellement à $10 \mu \mathrm{g} / \mathrm{l}$ à partir de 2014.

\section{L'eau chaude sanitaire}

Elle est produite par accumulation, semi-accumulation ou par production instantanée à partir de l'eau du réseau.

Elle est utilisée pour la toilette des patients, l'hygiène du personnel, le nettoyage des locaux.

Elle doit également respecter les normes de potabilité du décret $n^{\circ} 89-3$ modifié à l'exception de la température (qui peut dépasser $25^{\circ} \mathrm{C}$, sans excéder $60^{\circ} \mathrm{C}$ ) et de la concentration en aluminium (la valeur ne doit pas dépasser $0,5 \mathrm{mg} / \mathrm{l})$.

Lorsqu'un traitement complémentaire, tel que l'adoucissement est mis en œuvre; il ne doit concerner qu'une partie des eaux distribuées afin que le consommateur final puisse disposer d'une eau froide non soumise à ce traitement.

La responsabilité de l'eau d'alimentation à l'hôpital incombe au Directeur de l'établissement, qui est tenu non seulement de s'assurer de sa potabilité à l'arrivée mais aussi de faire procéder à des contrôles périodiques.

La production incombe à l'ingénieur, dont le choix est orienté par les moyens techniques et économiques à disposition (5).

Une mauvaise conception, une mauvaise mise en œuvre des installations, un entretien insuffisant ou le démantèlement intempestif de certaines installations, peuvent entraîner une dégradation de la qualité physico-chimique et/ou microbiologique de l'eau (7).

Pour prévenir l'entartrage et/ou la corrosion, divers traitements sont autorisés. Il peut s'agir de l'utilisation : (i) -de résines échangeuses de cations, (ii) - de réactifs à base de silicates et de polyphosphates alcalins, (iii) - de réactifs à base de polyphosphates alcalins et de sels de zinc et (iv) - de procédés à l'électrode soluble (aluminium et magnésium) (8). 
S'il y a bien une liste des traitements autorisés, il n'y a pas, à ce jour de liste des produits homologués, alors que le choix de ceux-ci n'est pas anodin (7).

Le contrôle en routine de la qualité physico-chimique de l'eau du réseau dans un établissement de santé comportera en plus de l'analyse de type $\mathrm{C} 1$ (odeur, saveur, couleur, turbidité, $\mathrm{pH}$, conductivité, chlore libre et total), les paramètres répertoriés dans le Tableau I.

La fréquence des prélèvements à la production est déterminée en fonction du débit journalier exprimé en $\mathrm{m}^{3} /$ jour (2).

Le type d'analyse à réaliser est fonction de l'emplacement:

- analyse de type C3 (analyse physico-chimique complète) et éventuellement des analyses particulières lorsqu'il s'agit de la ressource, c'est-à-dire du point de puisage avant traitement.

- analyse de type C3 ou C2 (analyse physico-chimique sommaire) et éventuellement des analyses particulières lorsqu'on s'adresse à la production, c'est-à-dire après traitement et avant refoulement dans le réseau.

- analyse de type $\mathrm{C} 1$ pour la distribution dans le réseau. L'analyse des échantillons d'eau prélevés est réalisée par des laboratoires agréés au titre du contrôle sanitaire des eaux destinées à la consommation humaine. Cet agrément est accordé par un arrêté du Ministre Chargé de l'Environnement.

Les conditions d'agrément d'un laboratoire concernent notamment la qualification des personnels, la nature des équipements dont il dispose et les méthodes d'analyses utilisées. Les méthodes d'analyse doivent être, soit des méthodes de référence, soit des méthodes conduisant à des résultats équivalents (9).

Les laboratoires sont donc autorisés à utiliser des tech- niques différentes à condition de démontrer l'obtention de performances identiques. En particulier, la fidélité et la justesse des deux méthodes sont déterminées et comparées. Il convient que de tels essais soient effectués sur les concentrations et les types d'échantillons appropriés (10).

\section{L'eau pour dilution des solutions concentrées pour hémodialyse}

Elle est produite à partir de l'eau du réseau après filtration, adoucissement, déminéralisation et osmose inverse.

Elle doit être conforme aux exigences de la Pharmacopée européenne (19 critères de qualité).

Les dispositions de la Pharmacopée sont données à titre d'information et de conseil et ne sont pas des normes opposables. Cependant les méthodes proposées par ce texte, constituent des éléments importants pour l'appréciation de la qualité de l'eau et serviront de référence (11).

Les teneurs en aluminium, en nitrates et en fluor de l'eau pour dilution des solutions concentrées pour hémodialyse sont plus basses que celles définies pour les eaux destinées à la consommation humaine. Ces valeurs sont respectivement de $10 \mu \mathrm{g} / \mathrm{l}$ au lieu de $200 \mu \mathrm{g} / \mathrm{l}$, pour l'aluminium ; de $2 \mathrm{mg} / \mathrm{l}$ au lieu de $50 \mathrm{mg} / \mathrm{l}$, pour les nitrates et de $0,2 \mathrm{mg} / \mathrm{l}$ au lieu de 0,7 à $1,5 \mathrm{mg} / \mathrm{l}$ (selon la température) pour le fluor. Cette eau est produite et utilisée en continu à raison d'une moyenne de 120 litres par séance de dialyse. Sa qualité n'est vérifiée qu'à postériori (12). La périodicité et le type d'analyse à effectuer sont fonction du nombre de séances de dialyse réalisées par année et par installation de traitement (13).

Tableau I : Paramètres à contrôler dans l'eau du réseau d'un établissement de santé.

\begin{tabular}{|l|l|l|l|}
\hline Paramètres & Expression du résultat & Niveau maximal admissible & Niveau guide \\
\hline Température & ${ }^{\circ} \mathrm{C}$ & 25 & \\
\hline Dureté & ${ }^{\circ} \mathrm{F}$ & - & 25 \\
\hline Phosphates & $\mathrm{mg} / \mathrm{l} \mathrm{en} \mathrm{P}_{2} \mathrm{O}_{5}$ & 5 & \\
\hline Silice & $\mathrm{mg} / \mathrm{l}$ & - & $<10$ si traitement par les silicates \\
\hline Aluminium & $\mathrm{mg} / \mathrm{l}$ & 0,2 & \\
\hline Magnésium & $\mathrm{mg} / 1$ & 50 & \\
\hline Zinc & $\mathrm{mg} / 1$ & 5 & \\
\hline Fer & $\mathrm{mg} / 1$ & 0,2 & \\
\hline Cuivre & $\mathrm{mg} / 1$ & 1 & \\
\hline Plomb & $\mu \mathrm{g} / 1$ & $\begin{array}{l}50 \text { (décembre 2003) puis } 25 \\
\text { (décembre 2013) puis } 10 \mu \mathrm{g} / \mathrm{l}\end{array}$ & \\
\hline
\end{tabular}




\section{Recueil des échantillons d'eau}

Toutes les eaux sont susceptibles de se modifier plus ou moins rapidement par suite de réactions physiques, chimiques ou biologiques pouvant se produire entre le prélèvement et l'analyse.

La nature et la vitesse de ces réactions sont souvent telles que, si les précautions nécessaires ne sont pas prises avant et pendant le transport ainsi qu'au cours de la conservation des échantillons au laboratoire avant analyse, les concentrations déterminées pourront être très différentes de ce qu'elles étaient au moment du prélèvement.

Les causes de ces variations sont nombreuses : (i) Certains composés (comme le fer ferreux) peuvent être oxydés par l'oxygène dissous, contenu dans les échantillons ou par l'oxygène de l'air, (ii) - Certaines substances peuvent précipiter [hydroxyde d'aluminium $\mathrm{Al}(\mathrm{OH})_{3}$ ] ou passer en phase vapeur (mercure) et (iii) les métaux dissous ou à l'état colloïdal peuvent être adsorbés ou absorbés de façon irréversible par la surface des récipients ou des matières solides contenues dans les échantillons.

L'importance de ces réactions est fonction de la nature chimique et biologique de l'échantillon, de sa température, de son exposition à la lumière, de la nature du contenant, du délai entre le prélèvement de l'analyse.

Les variations relatives à un constituant seront plus ou moins rapides et importantes en fonction du type d'eaux mais aussi en fonction des conditions saisonnières. Il est donc indispensable d'analyser les échantillons rapidement (10).

Quelques recommandations peuvent être formulées : (i) - remplir complètement les flacons et les boucher de manière à ce qu'il n'y ait pas d'air au-dessus de l'échantillon (le fer aura moins tendance à s'oxyder), (ii) - le récipient utilisé ne doit pas être cause de contamination. Il ne doit pas absorber ou adsorber les constituants à doser (des traces de métaux peuvent s'adsorber sur les parois d'un récipient en verre, ce qui peut être évité en acidifiant l'échantillon). Il est recommandé d'envisager des récipients à usage unique, afin d'éviter de contaminer des échantillons à faible teneur par des récipients ayant contenu des échantillons plus fortement dosés.

Des récipients en verre borosilicaté ou en plastique (PTFE, PVC, PET) peuvent être utilisés pour l'analyse des métaux suivants : argent, cadmium, chrome total, cuivre, fer total, manganèse, nickel, plomb et zinc.

Pour l'aluminium, un récipient en plastique et pour le mercure total, un flacon en verre borosilicaté sont plus appropriés. Certains éléments peuvent être stabilisés par l'ajout d'agents de préservation. Une acidification à $\mathrm{pH}$ inférieur à 2,0 permet pour les éléments métalliques précédemment cités de disposer d'une durée maximale de conservation d'un mois avant analyse.

Il convient de prendre en compte lors de l'analyse et du calcul des résultats, de toute dilution de l'échantillon par ajout de ces composés. Il est recommandé de prévoir la réalisation d'un essai à blanc notamment dans le cas de dosages des éléments à l'état de traces afin de tenir compte de l'apport éventuel par les agents de préservation d'une quantité supplémentaire des éléments à doser (par exemple les acides peuvent apporter une quantité non négligeable d'arsenic, de plomb, de mercure).

Pour le transport des échantillons, il est recommandé de les maintenir à une température aussi fraîche que possible, protégés de la lumière (10).

\section{Les méthodes de dosage}

Il s'agit essentiellement de méthodes spectrales.

\section{La spectrophotométrie}

Elle est utilisée pour le dosage du chrome VI (teneurs inférieures à $0.5 \mathrm{mg} / \mathrm{l}$ ) La réaction du chrome hexavalent avec la 1,5 diphénylcarbazide donne un complexe rouge-violet dont on mesure l'absorbance à une longueur d'onde de $540 \mathrm{~nm}$ (14).

Elle s'applique également au dosage du fer. Après solubilisation des composés du fer et réduction du fer III, il y a formation d'un complexe rouge-orangé en milieu tamponné entre le fer II et la phénanthroline. L'absorbance est ensuite mesurée à une longueur d'onde de $510 \mathrm{~nm}$ (15).

\section{Les méthodes recommandées par la Pharmacopée européenne reposent sur :}

* la spectrométrie d'absorption atomique pour le dosage du mercure et du zinc,

* la réalisation d'un essai limite pour l'aluminium et le plomb. Il consiste en la comparaison de l'échantillon à examiner à des solutions de références dont la préparation est décrite par la Pharmacopée (16).

\section{La spectrophotométrie d'absorption ato- mique en flamme}

Cette méthode est applicable au dosage des élémentsuivants : manganèse, fer, cobalt, nickel, cuivre, zinc, argent et plomb. Le dosage est direct lorsque les concentrations sont relativement élevées et qu'il n'y a pas d'interférences notables. Le dosage peut dans les autres cas se faire après complexation par le sel d'am- 
monium de l'acide pyrrolidinodithiocarboxylique1(APDC) suivie d'une extraction par la méthylisobutylcétone (MIBC) à pH 2,5 (17). Elle permet aussi le dosage du chrome total (teneurs de 0,5 à $20 \mathrm{mg} / \mathrm{l})(18)$, du cadmium (teneurs de $0,05 \mathrm{mg}$ à $1 \mathrm{mg} / \mathrm{l}$ ) (19) et de l'aluminium (20).

\section{La spectrophotométrie d'absorption ato- mique sans flamme}

Elle est utilisée pour le dosage du mercure (teneurs inférieures à $0,2 \mu \mathrm{g} / \mathrm{l})(21)$.

\section{La spectrométrie d'absorption atomique électrothermique}

Cette méthode s'applique au dosage de 15 éléments minéraux dont l'aluminium, l'argent, le cuivre, l'étain, le manganèse, nickel, plomb) (22) Elle est aussi utilisée pour le dosage du chrome total (18) et du cadmium (19).

\section{La spectrométrie de fluorescence ato- mique}

Elle s'applique au dosage du mercure total (teneurs de $0,01 \mu \mathrm{g} / \mathrm{l}$ à $20 \mu \mathrm{g} / \mathrm{l})$ après minéralisation par le brome (23).

\section{La torche à plasma avec détection par spectrométrie d'émission (ICP-AES)}

Le seul écueil de cette technique est lié aux interférences, lors du dosage d'éléments à l'état de traces. Deux grands types d'interférences peuvent se produire:

* des interférences liées au bruit de fond (lumière parasite),

* des interférences spectrales (recouvrement par la raie d'un autre élément, recouvrement non résolu d'un spectre de bande moléculaire).

Cette technique est applicable au dosage de 33 éléments dont l'aluminium, le cadmium, le chrome, le cuivre, le fer, le plomb, le manganèse, le nickel, l'argent, l'étain, le zinc (24).

\section{La torche à plasma avec détection par spectrométrie de masse}

Cette méthode permet aussi la détermination simultanée de plusieurs dizaines d'éléments (25).

\section{Expression des résultats et discussion}

La détermination de la concentration en l'élément à doser fait appel : soit à la méthode d'étalonnage exter- ne, soit à la méthode des ajouts dosés lorsque la méthode d'étalonnage externe ne permet pas d'obtenir des résultats suffisamment exacts en raison d'effets de matrice par exemple.

Les causes d'erreur possibles sont :

- les interférences (peu de méthodes analytiques sont totalement spécifiques pour les espèces à analyser),

- les effets de matrice (il peut s'agir de l'instabilité des échantillons entre le prélèvement et l'analyse ou de l'incapacité à déterminer toutes les formes pertinentes de l'espèce à analyser),

- l'essai à blanc et l'étalonnage biaisés (les échantillons et les étalons doivent être traités exactement de la même manière).

Le choix d'une méthode de dosage doit reposer sur la prise en compte des différents critères de qualité : exactitude, sensibilité, limite de détection,...

\section{Conclusion}

L'eau en milieu hospitalier lorsqu'elle n'est pas destinée à des usages particuliers tels que l'hémodialyse, doit être potable. Les matériaux constitutifs des canalisations et les traitements utilisés (coagulation, floculation) peuvent contribuer à élever les teneurs en métaux de l'eau.

L'aluminium fait toujours l'objet d'une surveillance particulière. Le nickel et le plomb voient leurs seuils abaissés par la directive européénne. Les métaux ne représentent qu'une partie des paramètres chimiques. Les pesticides et les hydrocarbures aromatiques peuvent également être recherchés.

La Direction de l'Hospitalisation et de l'Organisation des soins va lancer une mission d'évaluation sur l'utilisation de l'acier galvanisé pour les canalisations dans les hôpitaux.

La qualité de l'eau du réseau intérieur d'un établissement de santé ; c'est aussi le contrôle de la contamination microbienne. Des micro-organismes tels que les légionelles sont favorisés par la présence de résidus métalliques comme le fer, le cuivre ou le zinc. 


\section{Références}

1. AFIDTN-L'infirmière en néphrologie - Clinique pratique et évaluation de la qualité des soins - 1997 Masson.

2. Décret $n^{\circ} 89-3$ du 3 janvier 1989 relatif aux eaux destinées à la consommation humaine à l'exclusion des eaux minérales naturelles. Modifié par le décret n90-330 du 10 avril 1990, par le décret $\mathrm{n}^{\circ} 91-257$ du 7 mars 1991, par le décret $n^{\circ} 95-363$ du 5 avril 1995 et par le décret $n^{\circ} 98$ 1090 du 4 décembre 1998.

3. Directive 98/83/CE du Conseil du 3 novembre 1998 relative à la qualité des eaux destinées à la consommation humaine.

4. Hehn M. L'eau à l'hôpital - Hygiène en milieu hospitalier - $n^{\circ} 7$, avril 1998.

5. Mery D. Les eaux à l'hôpital et les champs de compétence et de responsabilité -XXèmes journées d'études de Pharmacie Hospitalière de l'APHIF - septembre 1998.

6. Berneron C. Qualité de l'eau pour hémodialyse : Bilan sur 18 mois au C.H.R.U de Lille-Mémoire du DES de Pharmacie Spécialisée - septembre 1999.

7. Groupe Eau et Santé- Eaux des établissements de santé Qualité de l'eau des réseaux intérieurs - décembre 2000. Réalisé avec le soutien du Laboratoire Asta Médica.

8. Circulaire DGS/VS4 $n^{\circ} 2000-166$ du 28 mars 2000 relative aux produits et procédés de traitement des eaux destinées à la consommation humaine.

9. AFNOR- La qualité de l'eau - Analyses biochimiques et biologiques - Analyses microbiologiquesRéglementation - Recueil Environnement.

10. AFNOR- La qualité de l'eau - Terminologie Echantillonnage - Contrôle qualité - Recueil Environnement.

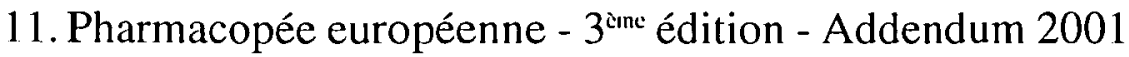
- Eau pour dilution des solutions concentrées pour hémodialyse.
12. Lusamvuku et coll.- Production d'eau pour hémodialyse Validation et démarche pour un fonctionnement en assurance qualité - J. Pharm. Clin. 18, n4 décembre 1999.

13. Circulaire DGS/DH/ASFSSAPSn ${ }^{\circ} 2000-337$ du 20 juin 2000 relative à la diffusion d'un guide pour la production d'eau pour l'hémodialyse des patients insuffisants rénaux.

14. Norme NFT 90-043- Essais des eaux - Dosage du chrome(VI)- octobre 1988 - Afnor.

15. Norme NFT 90-017- Essais des eaux- Dosage du ferjuin 1982-Afnor.

16. Pharmacopée européenne- $3^{\text {cinc }}$ édition - Addendum 2001 - Essais limites des impuretés inorganiques.

17. Norme FDT 90-112- Qualité de l'eau - Dosage de 8 éléments métalliques par spectrométrie d'absorption atomique dans la flamme -juillet 1998 - Afnor.

18. Norme NF EN 1233- Qualité de l'eau - Dosage du chrome - septembre 1996-Afnor

19. Norme NF EN ISO 5961- Qualité de l'eau - Dosage du cadmium par spectrométrie d'absorption atomique - août 1995 - Afnor.

20. Norme NF EN ISO 12020- Qualité de l'eau - Dosage de l'aluminium - juin 2000 - Afnor.

21. Norme NFT 90-131- Essais des eaux - Dosage du mercure total par spectrométrie d'absorption atomique sans flamme - septembre 1986 - Afnor.

22. Norme FDT 90-119- Qualité de l'eau - Dosage d'éléments minéraux - juillet 1998 - Afnor.

23. Norme XPT 90-113-2-Essais des eaux - Dosage du mercure total par spectrométrie de fluorescence atomiquefévrier 1997 - Afnor.

24. Norme NF EN ISO 11885- Qualité de l'eau - Dosage de 33 éléments par spectroscopie d'émission atomique avec plasma couplé par induction- mars 1998-Afnor.

25. Hamon et coll.- Méthodes spectrales et analyse orga-

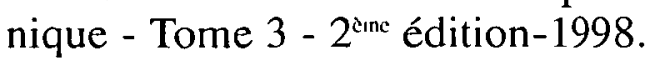

